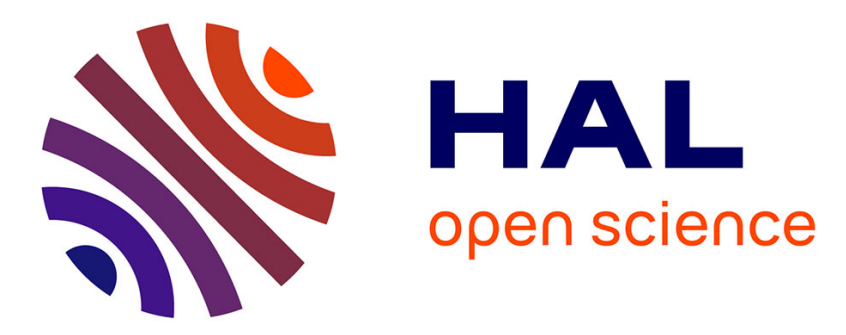

\title{
Flow of a yield-stress fluid over a cavity: experimental and numerical investigation of a viscoplastic boundary layer
}

\author{
Paul Vigneaux, Guillaume Chambon, Arthur Marly, Li-Hua Luu, Pierre
} Philippe

\section{To cite this version:}

Paul Vigneaux, Guillaume Chambon, Arthur Marly, Li-Hua Luu, Pierre Philippe. Flow of a yieldstress fluid over a cavity: experimental and numerical investigation of a viscoplastic boundary layer. Journal of Non-Newtonian Fluid Mechanics, 2018, 261, pp.38-49. 10.1016/j.jnnfm.2018.08.005 . hal01857303

\section{HAL Id: hal-01857303 https://hal.science/hal-01857303}

Submitted on 8 Oct 2018

HAL is a multi-disciplinary open access archive for the deposit and dissemination of scientific research documents, whether they are published or not. The documents may come from teaching and research institutions in France or abroad, or from public or private research centers.
L'archive ouverte pluridisciplinaire HAL, est destinée au dépôt et à la diffusion de documents scientifiques de niveau recherche, publiés ou non, émanant des établissements d'enseignement et de recherche français ou étrangers, des laboratoires publics ou privés. 


\title{
Flow of a yield-stress fluid over a cavity: experimental and numerical investigation of a viscoplastic boundary layer ${ }^{\vec{k}}$
}

\author{
Paul VIGNEAUX ${ }^{\mathrm{a}, \mathrm{b}, *}$, Guillaume CHAMBON ${ }^{\mathrm{c}, \mathrm{d}, *}$, Arthur MARLY, ${ }^{\mathrm{a}, \mathrm{b}}$, \\ Li-Hua LUU ${ }^{e}$, Pierre PHILIPPE ${ }^{\mathrm{e}}$ \\ ${ }^{a} U M P A, C N R S$ UMR 5669 ENS de Lyon, 46 allée d'Italie, F-69364 Lyon Cedex 07, \\ France \\ ${ }^{b}$ Univ Lyon, ENS de Lyon, INRIA NUMED Project-Team, 46 allée d'Italie, F-69364 \\ Lyon Cedex 07, France \\ ${ }^{c}$ Univ. Grenoble Alpes, IRSTEA, UR ETGR, 2 rue de la Papeterie, BP 76, F-38402 \\ Saint-Martin-d'Hères Cedex, France \\ ${ }^{d}$ OSUG, CNRS UMS 832, 122 rue de la Piscine, F-38400 Saint-Martin-d'Hères Cedex, \\ France \\ ${ }^{e} I R S T E A$, UR RECOVER, 3275 route de Cézanne, F-13182 Aix-en-Provence, France
}

\begin{abstract}
The ability of viscoplastic fluids to self-select their flow geometry through the formation of unyielded dead zones has important consequences for flows in wavy channels, flows over obstacles, etc. Yet, the mechanisms controlling the formation and dimensions of the dead zones remain poorly understood. We present a detailed cross-comparison of experimental and numerical results concerning channel flows of a viscoplastic fluid over a rectangular cavity filled by the same material. In all the configurations studied, which correspond to moderate values of the Bingham number, a continuous dead zone forms inside the cavity. Both numerical and experimental data reveal that, unlike at high Bingham numbers, the shear-rate profiles above the dead zone display an asymmetric shape. Accordingly, two different flow zones can be distin-
\end{abstract}

\footnotetext{
"Funded by the CNRS Interdisciplinary program "Défi InFIniti" (2017 and 2018). This article was published in 2018, Vol. 261, p 38-49 with DOI link: 10.1016/j.jnnfm.2018.08.005

${ }^{*}$ Corresponding authors

Email addresses: Paul.Vigneaux@math.cnrs.fr (Paul VIGNEAUX), guillaume.chambon@irstea.fr (Guillaume CHAMBON)
}

Preprint submitted to J. of Non-Newton. Fluid Mech. - Authors' version on HAL. October 8, 2018 
guished: a Poiseuille-like zone, in which the velocity profile is similar to that over a rigid wall, and a boundary layer ensuring the transition with the dead zone below. It is shown that the effective boundary condition felt by the Poiseuille-like layer is essentially controlled by incoming flow characteristics, such that the thickness of this layer does not obey simple relations with cavity length. Interestingly, however, the thickness of the boundary layer appears to follow a generalized Oldroyd's scaling with cavity length.

Keywords: Bingham rheology, Herschel-Bulkley rheology, viscoplastic boundary layer, extended Oldroyd scaling, particle image velocimetry, numerical simulations, Augmented Lagrangian method

\section{Contents}

1 Introduction 2

2 Methods 5

2.1 Experimental setup . . . . . . . . . . . . . 5

5 2.2 Numerical simulations . . . . . . . . . . . . . . 6

2.3 Fluid rheological parameters and flow regimes . . . . . . . 7

3 First flow features by cross-comparison experiments/simulations

3.1 Stress fields and dead zone . . . . . . . . . . . . . . . . . 9 9

3.2 Flow zones . . . . . . . . . . . . . . . . . . . 11

$10 \quad 3.3$ Dead zone interface . . . . . . . . . . . . . . . . 14

3.4 Flow characteristics at the boundary layer interface . . . . . . 15

3.5 First conclusions . . . . . . . . . . . . . . 16

4 Extended scaling for the boundary layer 18

4.1 Boundary layer equation . . . . . . . . . . . . . . . 18

$15 \quad 4.2$ Generalized scaling for boundary layer thickness . . . . . . . . 20

5 Final remarks $\quad 23$

\section{Introduction}

In this article, we study the flow of viscoplastic fluids in expansion - contraction confined domains. A striking feature of such configurations is the 
self-selection of the flowing region through the formation of fully unyielded dead zones and moving plugs [1]. Detailed numerical investigations have shown that various topologies of unyielded zones can occur, depending on incoming flow and channel characteristics [2, 3, 4]. However, understanding the mechanisms that govern the location of the yield interfaces remains an open issue. In real yield-stress fluids, further complexity can also be introduced by additional rheological trends of the materials, such as viscoelasticity or thixotropy, that may also play an influence on the formation of unyielded zones $[5,6]$. In this article, we want to shed light on the flow properties in the vicinity of the yield interfaces, with the objective to exhibit possible generic scaling relations. We consider more specifically the configuration of a longitudinal parallel flow over a rectangular cavity $[7,8]$ and, through detailed cross-comparisons between numerical simulations and physical experiments, focus on features induced by genuine viscoplasticity only.

Depending on whether numerical or experimental results are concerned, we will consider the Bingham or the Herschel-Bulkley (HB) constitutive law for the deviatoric stress tensor $\tau$ :

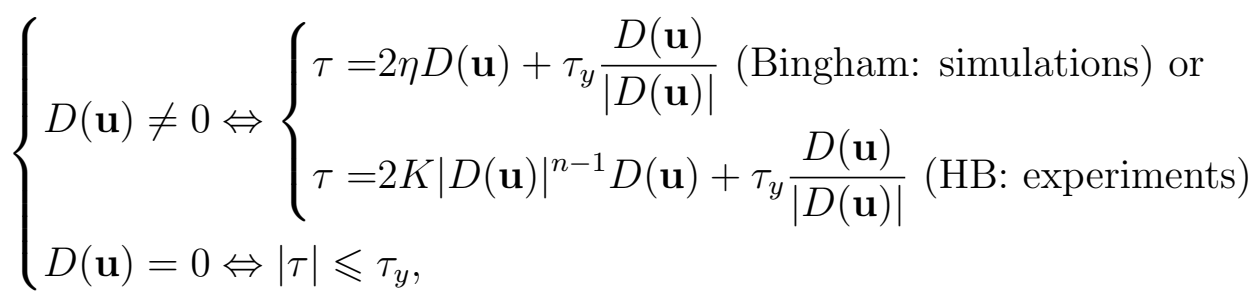

where $\tau_{y}$ is the yield stress (in $\mathrm{Pa}$ ), $\eta$ and $K$ are respectively the Bingham viscosity and $\mathrm{HB}$ consistency (resp. in $\mathrm{Pa} \cdot \mathrm{s}$ and $\mathrm{Pa} \cdot \mathrm{s}^{n}$ ), $n$ is a power-law index, $\mathbf{u}$ is the velocity, and $D(\mathbf{u})=\left(\nabla \mathbf{u}+\nabla \mathbf{u}^{T}\right) / 2$ is the strain rate tensor. We adopt the following convention for the tensor norms:

$$
|T|^{2}=\frac{1}{2} \sum_{i, j} T_{i, j}^{2}
$$

Clearly, Bingham law corresponds to a particular case of Herschel-Bulkley law for $n=1$ (and $K=\eta$ ). In the following, we assume that the Reynolds number $(R e)$ is low, so that the flows are only controlled either by the Bingham $B$ or by the Herschel-Bulkley number $H b$, commonly referred to as viscoplastic numbers, defined as:

$$
B=\frac{\tau_{y}}{\eta} \frac{\mathcal{L}}{\mathcal{U}} \text { and } H b=\frac{\tau_{y}}{K}\left(\frac{\mathcal{L}}{\mathcal{U}}\right)^{n},
$$


where $\mathcal{L}$ and $\mathcal{U}$ denote characteristic length and velocity scales, respectively. 35 Note that various choices for the characteristic scales $\mathcal{L}$ and $\mathcal{U}$ will be considered in the following, leading to different definitions of the viscoplastic numbers $B$ and $H b$ (specified by different subscripts).

Recently, compelling analytical predictions for the location of yield interfaces in viscoplastic flows over a cavity were obtained by Balmforth et al. [4] in the limit of high Bingham numbers. In this regime, these authors show that the sheared flow layer developing between the unyielded zones behaves as a viscoplastic boundary layer. The theory of viscoplastic boundary layers goes back to Oldroyd [9], who studied analytically different flow configurations including a jet intrusion of Bingham fluid in an infinite half-plane and a moving knife in an infinite plane. Oldroyd derived an asymptotic approximation of the momentum conservation equation for high Bingham numbers, and obtained similarity solutions for the velocity profile in the yielded zones, dubbed boundary layers. The width of these boundary layers evolves according to $B_{\ell}^{-1 / 3} \ell$, where $\ell$ denotes the abscissa and $B_{\ell}$ corresponds here to a steal Bingham number defined on the basis of $\ell$. This problem has then been studied in other configurations, e.g. the flow around an obstacle where an alternative scaling in $\mathcal{O}\left(B^{-1 / 2}\right)$ was proposed [10], or in a Hele-Shaw cell [11]. Through asymptotic analysis and numerical simulations, Balmforth et al. [4] revisited Oldroyd's viscoplastic boundary layer theory in various configurations and provided a rationalization of the apparently incompatible scalings previously proposed. In short, both scalings in $\mathcal{O}\left(B^{-1 / 3}\right)$ and $\mathcal{O}\left(B^{-1 / 2}\right)$ can occur, depending on whether the zone bounding the viscoplastic boundary layer is an unyielded layer or a wall. In the case of the flow over a cavity, they showed that the thickness of the boundary layer obeys Oldroyd's scal${ }_{60}$ ing in $B_{D}^{-1 / 3} D$, where $D$ denotes the length of the cavity. Noteworthy for the following is the fact that Balmforth et al.'s analysis assumes shear-rate profiles in the flow layer that are symmetrical with respect to the center of the layer.

In this paper, we investigate whether the results of [4] concerning the case of cavity flow can be extended to the case of moderate viscoplastic numbers, i.e. outside of the a priori domain of validity of the asymptotic approximation but for a range of parameters that is more relevant of applications. The work is based on a re-analysis of the experimental results described in [8] through comparisons with highly-resolved numerical simulations. Section 2

70 briefly recalls the experimental and numerical methods, which are described in full details in [8] and [3], respectively. In section 3, we highlight the 
common features and trends observed in experiments and simulations, and show that the location of the yield interfaces cannot be predicted through Oldroyd's scaling in our case. Section 4 shows that a sub-layer of the shear zone does nevertheless behave as a viscoplastic boundary layer, and that the thickness of this layer indeed obeys a generalized Oldroyd's scaling in both simulations and experiments. Lastly, section 5 presents final remarks.

\section{Methods}

\subsection{Experimental setup}

(a)
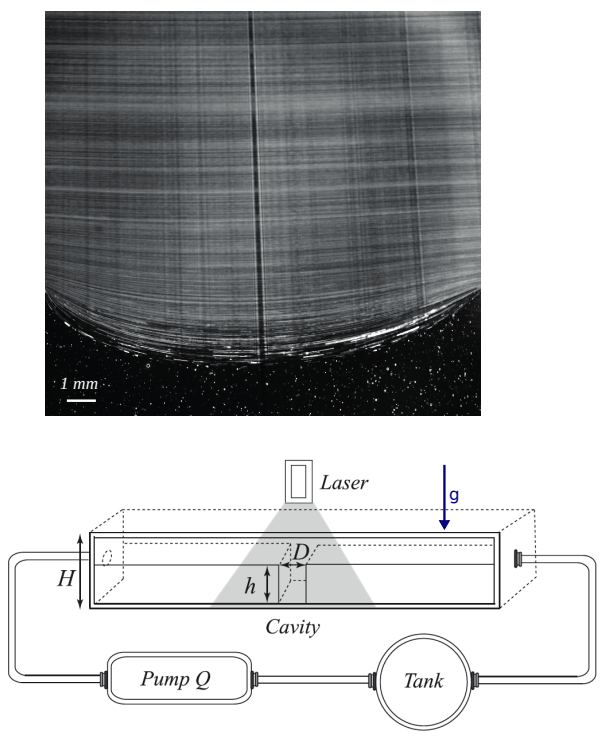

(b)

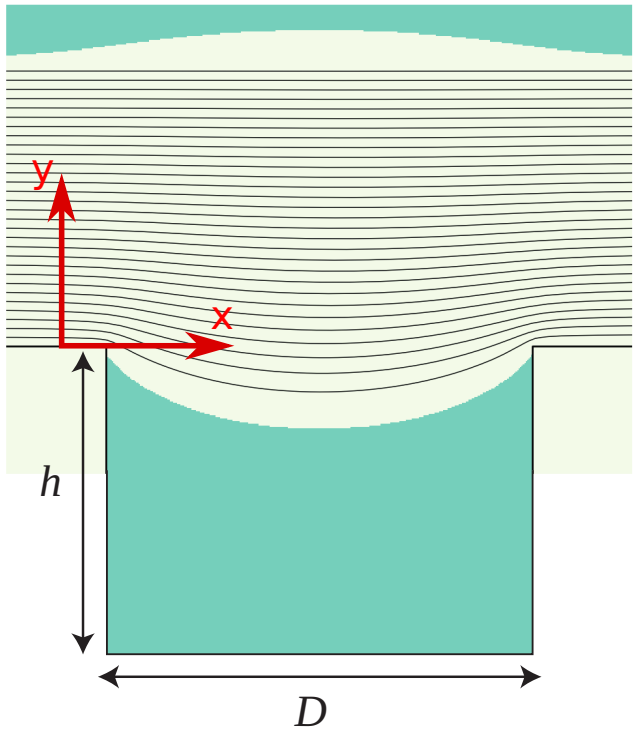

Figure 1: (a) Experimental setup (bottom) and streamlines for an experiment with $D=$ $1.5 \mathrm{~cm}, h=3 \mathrm{~cm}$, fluid $\mathrm{F} 3$ and flow rate $Q=6.4 \mathrm{~cm}^{3} / \mathrm{s}$. The pattern of streamlines is visualized by computing the standard deviation of local pixel gray levels over a 1-minute image sequence (2723 images). (b) Numerical simulation for the same geometry and fluid, for $Q=8 \mathrm{~cm}^{3} / \mathrm{s}$. The unyielded plug and dead zones are colored darker. We also sketch the location of the coordinate system, with $y=0$, corresponding to cavity top.

The experimental setup was described in [8]. As shown in Figure 1(a), it consists of a closed rectangular channel with a height $H=6.5 \mathrm{~cm}$, a length of $30 \mathrm{~cm}$, and a width of $8 \mathrm{~cm}$. Two steps are placed in the cell to form a 
central cavity of height $h$ (equal to 1.5 or $3 \mathrm{~cm}$ ). The cavity length $D$ is equal to $1.5,3$ or $6 \mathrm{~cm}$. In the following, the $x$ axis corresponds to the longitudinal direction of the cell, while $y$ denotes the cross-stream vertical direction. Note that $y$ is oriented upwards, with $y=0$ corresponding to the top of the cavity.

Stationary laminar flows are generated by connecting the cell to a pump imposing a constant flow rate $Q$. We use a transparent model yield-stress fluid, namely Carbopol micro-gel solutions, whose rheological behaviour is well described, in steady state, by a Herschel-Bulkley law [12, 13]. Note however that this material also features viscoelastic properties that can induce additional complexity to the flows $[14,7]$.

Using reflective tracers (hollow spheres) illuminated with a laser sheet, velocity fields $\mathbf{u}$ within the flow are reconstructed through particle image velocimetry $(\mathrm{PIV})$. The local shear rate $D(\mathbf{u})$ is computed by direct differentiation of the velocity field, and stress fields are then derived by application of Herschel-Bulkley constitutive law. Figure 1(a) presents a visualization of the streamlines obtained in a typical experiment. Note that in the vicinity of the cavity center (denoted by $x=x_{1 / 2}$ in the following), the flow can be considered as quasi-longitudinal, i.e. vertical velocity components are negligible. We can therefore spatially average PIV measurements in this region, to obtain cross-section profiles of longitudinal velocity with a higher accuracy. For more details, we refer to $[15,8]$.

\subsection{Numerical simulations}

As already mentioned, we assume $R e \ll 1$ and neglect inertia terms. We thus solve numerically the following viscoplastic Stokes system (where $p$ denotes a modified fluid pressure accounting for the hydrostatic contribution):

$$
\left\{\begin{array}{l}
\partial_{x} p=\partial_{x} \tau_{x x}+\partial_{y} \tau_{x y} \\
\partial_{y} p=\partial_{x} \tau_{x y}+\partial_{y} \tau_{y y} \\
\operatorname{div}(\mathbf{u})=0
\end{array}\right.
$$

A Bingham rheology is considered in the simulations. The computations are performed with a parallel 2D code (implemented with F90 and MUMPS (MPI)) based on Augmented Lagrangian methods. It shall be noted that fine meshes are used, together with demanding convergence criteria of the Augmented Lagrangian iterations, leading to very well defined and sharp localization of the yielded / unyielded material interfaces. As a consequence, strain rates and stresses are computed with high accuracy. For more details, 
we refer to [3], where the code was validated and compared to the physical experiments of [16] and [15]. We recall that the simulated configuration is a full expansion-contraction, so that the flow conditions on the upper half of the domain are not exactly the same in the experiments (plane upper wall as shown in Fig. 1(a, down)) and in the simulations (symmetric cavity facing downwards; this full geometry is shown in [3]-Fig. 2)). It was checked that, in spite of this difference, the same bottom-half analytic viscoplastic Poiseuille profiles are recovered in both the entry and exit channels (see [3] for more details). It was also shown that the simulations are able to retrieve several non-trivial qualitative features of the experiments. In particular, as illustrated in Figure 1b, the overall simulated flow pattern in the bottom-half of the domain above the cavity indeed appears in good qualitative agreement with the experimental observations. Based on these first comparisons, we argue that the differences in rheological model and geometrical configuration between the simulations and the experiments shall not constitute an obstacle to the comparison of both data sets, at least as long as the focus remains on main trends and qualitative features.

\subsection{Fluid rheological parameters and flow regimes}

The Herschel-Bulkley rheological parameters of the five Carbopol samples used in the physical experiments are recalled in Table 1 . Note that while the yield stress $\tau_{y}$ and consistency $K$ of the different fluids vary, the exponent $n$ remains close to 0.4 in all cases. As the numerical simulations are performed with a Bingham model, we adapted the rheological parameters considered in the computations to obtain comparable flow configurations. To do so, for a given experiment, we needed to adjust two parameters in the code, namely the Bingham viscosity $\eta$ and the "driving pressure" term. These two parameters are fixed through the two following constraints: (i) the plug velocity (i.e. the maximum velocity) in the center of the channel is the same as in the experiment, and (ii) the viscosity $\eta$ is computed from $K$ and the estimated maximal strain rate in the middle of the cavity (at $x=x_{1 / 2}$ ) obtained in the experiment. The yield stress $\tau_{y}$ is taken identical to the experimental value. These adjustments guarantee that shear-rate values are on the same order in both simulations and experiments (within a factor of 3 , typically), and that the two underlying constitutive laws are reasonably close to each other in the considered shear-rate range.

For the sake of clarity, Table 2 summarizes the different definitions of Bingham and Herschel-Bulkley viscoplastic numbers that will be used in the 


\begin{tabular}{lllll}
\hline Symbol & Fluid & $\tau_{y}(\mathrm{~Pa})$ & $\mathrm{K}\left(\mathrm{Pa} \cdot \mathrm{s}^{n}\right)$ & $n$ \\
\hline$\downarrow$ & F1 & 0.2 & 1.1 & 0.45 \\
$\triangle$ & F2 & 0.8 & 1.4 & 0.44 \\
$\bigcirc$ & F3 & 3.8 & 3.0 & 0.41 \\
$\square$ & F4 & 15.3 & 7.1 & 0.40 \\
$\diamond$ & F5 & 21.3 & 9.6 & 0.40 \\
\hline
\end{tabular}

Table 1: Herschel-Bulkley rheological parameters for the five Carbopol samples used in the experiments: yield stress $\tau_{y}$, consistency $K$, power index $n$. The symbols indicate the glyphs used in all subsequent figures.

\begin{tabular}{|l}
$\square$ \\
$D=1.5 \mathrm{~cm}, h=1.5 \mathrm{~cm}$ \\
$\square D=1.5 \mathrm{~cm}, h=3 \mathrm{~cm}$ \\
$\square D=3 \mathrm{~cm}, h=1.5 \mathrm{~cm}$ \\
$\square D=3 \mathrm{~cm}, h=3 \mathrm{~cm}$ \\
$\square D=6 \mathrm{~cm}, h=1.5 \mathrm{~cm}$ \\
$\square D=6 \mathrm{~cm}, h=3 \mathrm{~cm}$
\end{tabular}

Figure 2: Colors legend used, in the following figures of the article, for the various geometries of the cavity.

following. The numbers $B_{u p}$ and $H b_{u p}$, defined on the basis of maximum flow 150 velocity $U_{u p}$ at cavity entrance and upward channel width $(H-h) / 2$, characterize the incoming viscoplastic flow upward of the cavity. The numbers $B_{D}$ and $H b_{D}$, defined on the basis of $U_{u p}$ and cavity length $D$, correspond to those involved in classical Oldroyd's scaling. Lastly, we will also introduce the numbers $B_{c a v}$ and $H b_{c a v}$ based on an internal flow velocity above the cavity $U_{s}$ and cavity length $D$ (see section 4.2). The configurations investigated in this study correspond to moderate values of the upward viscoplastic numbers $B_{u p}$ and $H b_{u p}$ : the range of $H b_{u p}$ investigated in the experiments is $0.2-5$, while the corresponding range of $B_{u p}$ in the simulations is $0.5-40$.

Table 2: Characteristic length and velocity scales $\mathcal{L}$ and $\mathcal{U}$ (see Eq. (2)) used to define the different Bingham and Herschel-Bulkley viscoplastic numbers introduced in this study.

\begin{tabular}{lll}
\hline Simul./Exp. & $\mathcal{L}$ & $\mathcal{U}$ \\
\hline$B_{\text {up }} / H b_{u p}$ & $(H-h) / 2$ & $U_{u p}$ \\
$B_{D} / H b_{D}$ & $D$ & $U_{u p}$ \\
$B_{\text {cav }} / H b_{\text {cav }}$ & $D$ & $U_{s}$ \\
\hline
\end{tabular}




\section{First flow features by cross-comparison experiments/simulations}

160 observed in both experiments and numerical simulations. We focus in particular on the geometry of the dead zone in the cavity and a so-called boundary layer in the yielded zone.

\subsection{Stress fields and dead zone}

165 sical stationary viscoplastic Poiseuille flow [8, 3]. Hence, shear stress is maximum at the wall, and linearly decreases away from it. An unyielded moving plug is present in the center of the channel.

Figures 3 and 4 show the typical patterns of stress fields and unyielded tal data, the similarities between the results obtained in simulations and in experiments are evident. In both cases, the shear stress $\tau_{x y}$ presents a maximum that generally follows a slightly curved path close to the top of the cavity (indicated by the black curve in Fig. 3), while the normal stress $\tau_{x x}$ lar, the sign of $\partial_{x} \tau_{x x}$ is opposite in the two regions. A continuous unyielded plug is observed in the center of the channel, while a static unyielded dead zone forms at the bottom of the cavity. In the simulations, thanks to the high accuracy of the numerical scheme, the two yield surfaces, denoted by the plug), are easily identified as the positions where the values of strain rate vanish. In experiments, the location of the dead zone $y_{\text {int }}(x)$ can be determined precisely from the images (see Fig. 1). The determination of the upper plug $y_{\text {plug }}(x)$ is more difficult, as it is strongly sensitive to the errors on ity center $x_{1 / 2}$ (through a fit of the longitudinally-averaged velocity profile), such that the position of the upper plug in Figure 4 is only indicative.

To compare quantitatively the shape of the dead zone formed in the cavity in simulations and in experiments, we computed the shape factor $\Phi=A_{\text {tot }} /\left(D\left|y_{\text {int }}\left(x_{1 / 2}\right)\right|\right)$, where $A_{\text {tot }}=\int_{0}^{D}\left|y_{\text {int }}(x)\right| \mathrm{d} x$ is the area of yielded material in the cavity and $y_{\text {int }}\left(x_{1 / 2}\right)$ corresponds to the deepest position of the solid-liquid interface. As shown in Figure 5, it is observed that $\Phi$ is essentially constant for all the configurations studied, in both experiments and simulations. This is indicative of a shape-invariance property of the yield 


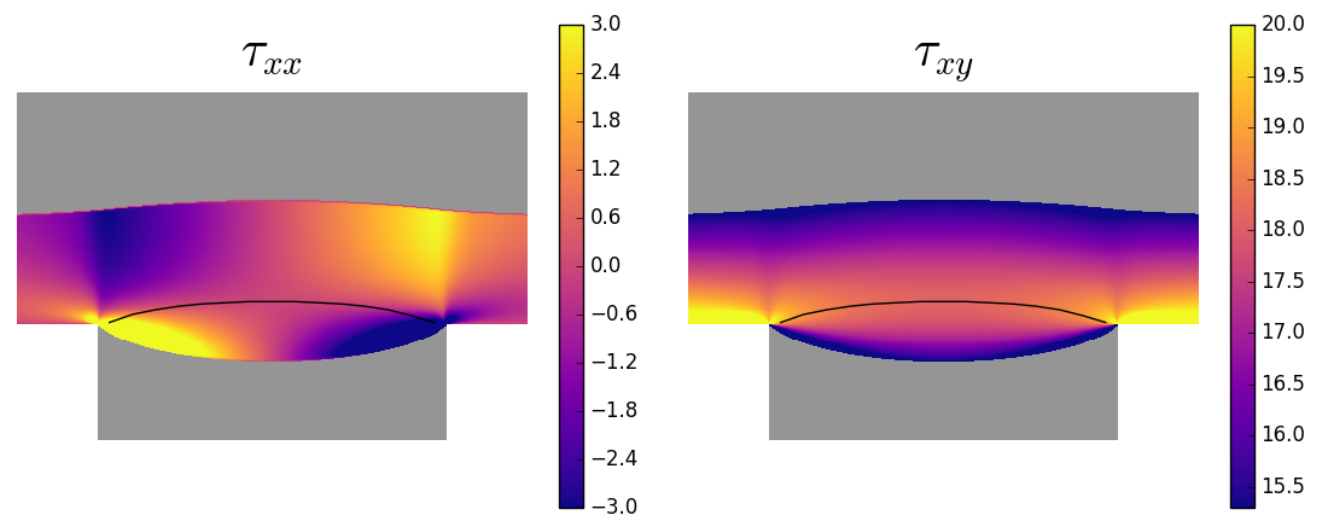

Figure 3: Numerical simulations: colormaps of normal and shear stress $\tau_{x x}$ and $\tau_{x y}$ (case $D=1.5 \mathrm{~cm}, h=3 \mathrm{~cm}$ for fluid F4). Colorbar values in Pa. The thick black curve represents the location of the maximum of $\tau_{x y}$ or, equivalently, the upper limit of the boundary layer $y_{s}(x)$ (see text). The gray areas correspond to the unyielded zones. Note that the maps represent a zoom-in view of the viscoplastic boundary layer above the cavity. The lower and upper channel walls are not shown.

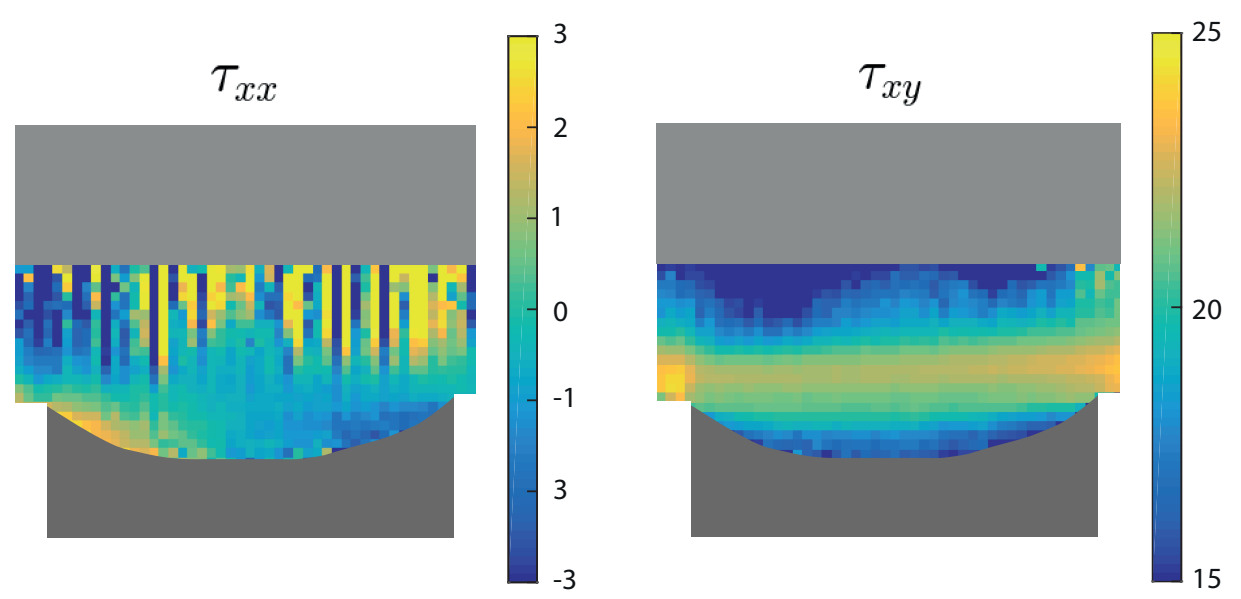

Figure 4: Experiments: same as Fig. 3 (case $D=1.5 \mathrm{~cm}, h=3 \mathrm{~cm}$ with fluid F4 and a flow rate $Q=15.3 \mathrm{~cm}^{3} / \mathrm{s}$ ). The upper unyielded zone has been traced on the basis of the determination of $y_{\text {plug }}$ at cavity center $x_{1 / 2}$ and is only indicative. As in Fig. 3 , the maps represent a zoomed-view of the viscoplastic boundary layer above the cavity.

interface, independently of cavity length and depth. Note that this result is necessarily restricted to the case of relatively small cavity lengths, for which 
the yield interface does not reach cavity bottom. For very long cavities, we expect $y_{\text {int }}\left(x_{1 / 2}\right) \rightarrow h$, and thus $\Phi \rightarrow 1$. In addition, it is observed that the values of $\Phi$ are comprised within the same range $(0.7-0.8$ typically) in numerical and experimental results (Fig. 5), thereby showing the similarity of the interface shape in both cases.
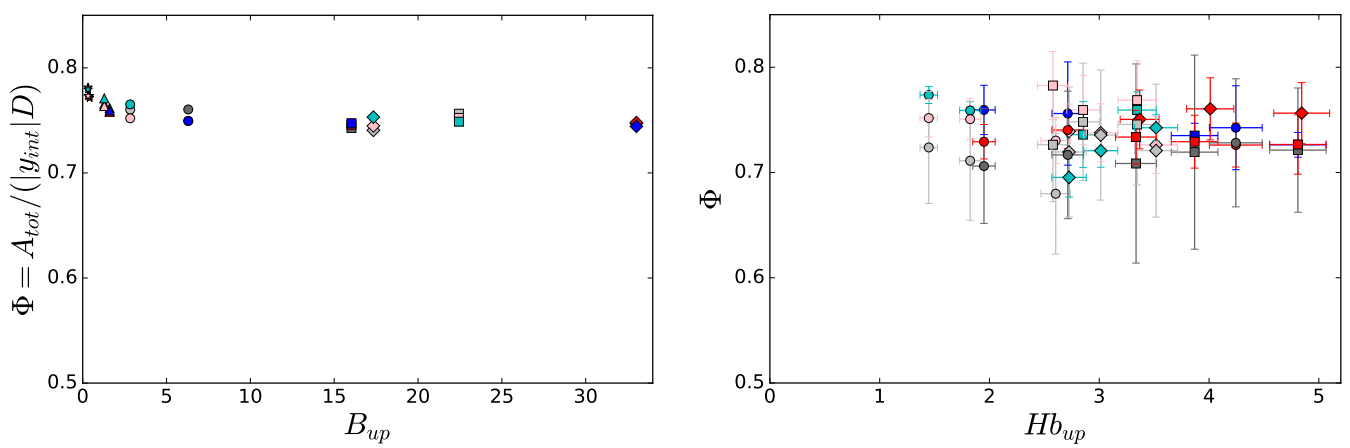

Figure 5: Shape factor of the solid-liquid interface $\Phi$ (see definition in text) as a function of viscoplastic numbers $B_{u p}$ or $H b_{u p}$. Glyphs correspond to Table 1, while colors legend are indicated in Figure 2. Left: simulations. Right: experiments.

\subsection{Flow zones}

Figure 6 presents typical profiles of longitudinal velocity $u$ and shear rate $|D(\mathbf{u})|$ at $x=x_{1 / 2}$ in the simulations. Note that $|D(\mathbf{u})|=\left|\partial_{y} u\right|$ at this location. By symmetry, only the first half of the profiles is shown. Qualitatively, these profiles were found to display similar features in all the simulations performed. The velocity profile shows a characteristic sigmoidal shape, increasing from 0 at the interface with the lower dead zone to a maximum in the upper plug zone. The shear-rate profile is bell-shaped, with a well-defined maximum in between. Remark that the profile is generally non-symmetric with respect to its maximum. The location of the shear rate maximum is denoted by $y_{s}$. Similarly, to simplify the notations, the positions of the lower and upper yield surface at $x_{1 / 2}$ will henceforth be denoted by $y_{\text {int }}$ and $y_{\text {plug }}$, respectively (Fig. 6). Lastly, the velocity at $\left(x_{1 / 2}, y_{s}\right)$ is denoted by $U_{s}{ }^{1}$.

\footnotetext{
${ }^{1}$ The $s$ index is reminiscent of the "slip" nomenclature used in [15]. We remind that this nomenclature is not related to the slipline theory for plastic solids (as described in $[17])$.
} 

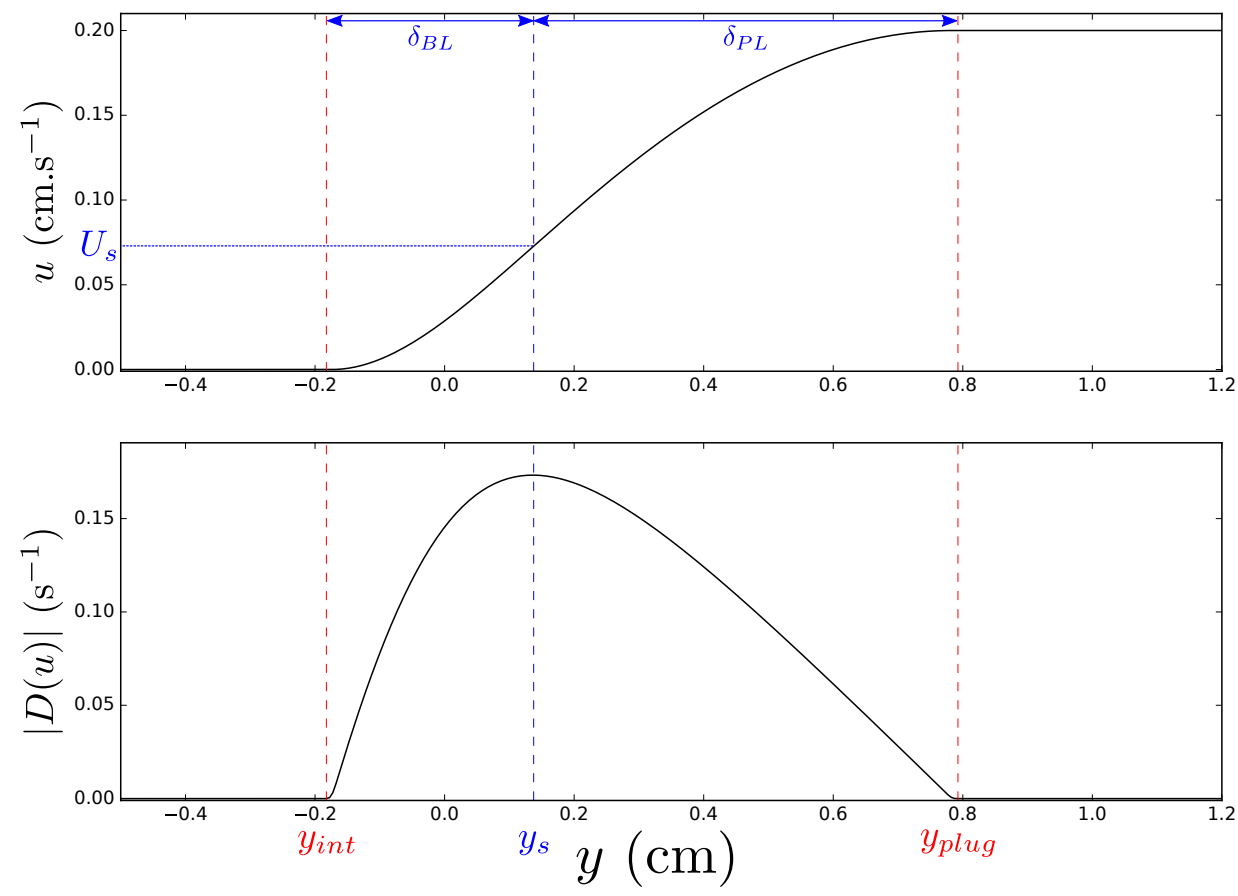

Figure 6: Numerical simulations: Typical cross-section profiles of longitudinal velocity $u$ (top, in $\mathrm{cm} . \mathrm{s}^{-1}$ ) and shear rate $|D(\mathbf{u})|$ (bottom, in $\mathrm{s}^{-1}$ ) at $x=x_{1 / 2}$, the middle of the cavity ( $y$ in $\mathrm{m}$ ) (fluid F3, same parameters as in Fig. 7). By symmetry (see section 2.2 for details on the geometrical configuration of the simulations), only the lower halves of the profiles are shown. Note the sharpness of $|D(u)|$ at the interfaces between the yielded and unyielded zones, indicative of the high-quality numerical resolution.

Based on these profiles and the observation of stress fields (see section 3.1), we distinguish between three different zones in the flow:

- a zone of width $\delta_{B L}$ between $y_{i n t}$ and $y_{s}$, which we will refer to as the boundary layer,

- a zone of width $\delta_{P L}$ between $y_{s}$ and $y_{p l u g}$, which we will refer to as the Poiseuille-like layer,

- the moving plug zone above $y=y_{\text {plug }}$.

Note that the definition of boundary layer introduced here is slightly different from that used in $[9,4,16]$, where this terminology referred to the whole yielded zone. Instead, as will be discussed later, we found it relevant in our 
case to limit the boundary layer to the zone in which the shear rate, and consequently the shear stress, increase. The terminology of Poiseuille-like layer for the stress-decrease zone situated above, comes from the observation of a quasi-linear decrease of shear stress in this zone, exactly as in the incoming Poiseuille flow upstream of the cavity [8].
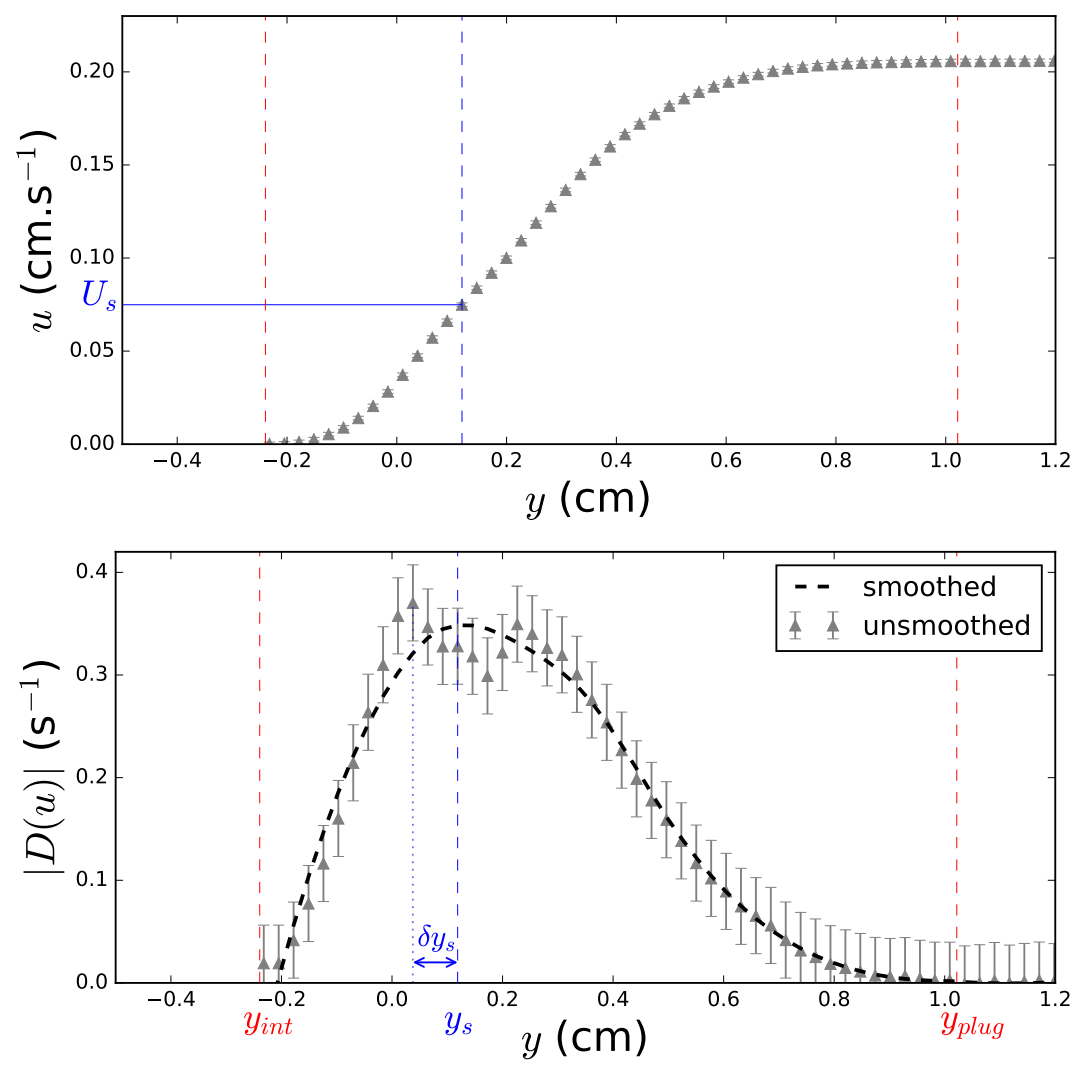

Figure 7: Experiments: Typical vertical profiles of longitudinal velocity $u$ (top) and shear rate $|D(u)|$ (bottom) at $x=x_{1 / 2}$, the middle of the cavity (fluid F3 with $D=1.5 \mathrm{~cm}$, $h=1.5 \mathrm{~cm}$ and $Q=6.4 \mathrm{~cm} \cdot \mathrm{s}^{-1}$, like in Fig. 6). The location $y_{s}$ of the maximum of $|D(u)|$ is determined on the smoothed profile (dashed curve, see text), while the corresponding uncertainty $\delta y_{s}$ accounts for the difference with the maximum of the unsmoothed values (symbols).

As shown in Figure 7, velocity and shear-rate profiles at $x=x_{1 / 2}$ obtained in the experiments present similar shapes, such that similar flow zones can be defined. When the shear rate is computed from measured velocity profiles through a simple finite-difference scheme, the peak in the shear rate profile 
is sometimes less marked than in the simulations, with the existence of an 235 apparent plateau around the maximum (well visible in Fig. 7). However, if a smoothing procedure is applied on velocity profiles prior to computing the derivative ( $3^{\text {rd }}$-order polynomial fit over moving windows of 21 points), a single shear rate maximum is retrieved. Observe that the smoothed shearrate profile remains consistent with unsmoothed data points if error bars are taken into account. As explained in [8], plateaus in unsmoothed shear-rate profiles were generally observed for the largest cavity lengths $D$. Whether these plateaus represent a true physical feature, or are due to measurement errors, remains to be elucidated. In [8] possible correlations between the width of these plateaus and viscoelastic rheological properties of Carbopol were investigated, but no definitive conclusion could be drawn. In the present study, since the numerical simulations (which account for a genuine viscoplastic rheology) never exhibit such plateaus, we decided to base our analyses of experimental data on the smoothed shear-rate profiles. This allows us to uniquely determine $y_{s}$ and $U_{s}$ (Fig. 7) and directly compare these quantities to their numerical counterparts. Error bars associated to these quantities are determined to reflect the differences with the non-smoothed case (and hence the robustness of the values with respect to the smoothing procedure adopted).

Finally we note that even though later analyses will mostly focus on quantities defined at $x_{1 / 2}$, the zoning introduced above can be defined along the whole length of the cavity. In numerical simulations, the position $y=y_{s}(x)$ of maximum shear rate, or equivalently of maximum shear stress, can be determined for all values of $x$ (accounting in this case for the complete expression of $|D(\mathbf{u})|$ ). A typical example is shown in Figure 3 (see also Fig. 14).

260 The boundary layer, comprised between $y_{\text {int }}(x)$ and $y_{s}(x)$, generally presents a lentil shape, with a thickness progressively vanishing at cavity edges. Interesting also is that $y_{\text {plug }}(x)$ seems to follow the shape of the boundary layer. In experiments, the precise determination of $y_{s}(x)$ all along the cavity is more difficult, due to the relatively large noise level on $|D(\mathbf{u})|$-values as soon as spatial averaging cannot be applied. Remark nevertheless that the overall shape of the boundary layer appears qualitatively similar to that observed in the simulations (see Fig. 4).

\subsection{Dead zone interface}

Figure 8 shows the evolution of dead zone interface depth $y_{\text {int }}$ in simulations and in experiments. In both cases, it is observed that $y_{\text {int }}$ significantly 
decreases with cavity length $D$ (Fig. 8 - insets). Furthermore, as shown in the main panels, the ratio $\left|y_{\text {int }}\right| / D$ appears to roughly follow power-law trends with the viscoplastic numbers $B_{D}$ or $H b_{D}$ defined on the basis of incoming flow velocity $U_{u p}$ and cavity length $D$ (see Tab. 2). In the simulations, the trend is consistent with Oldroyd's scaling, namely $\left|y_{\text {int }}\right| / D \propto B_{D}^{-1 / 3}$. Similarly, in experiments, the power-law trend is consistent with an exponent of $-1 /(n+2)$ corresponding to the Herschel-Bulkley version of Oldroyd's scaling [4]. We remark nevertheless that the dispersion of data points around these main trends remains significant, particularly in the experimental case. Even in numerical results, variations of $\left|y_{\text {int }}\right| / D$ of more than $50 \%$ can be observed for a given value of $B_{D}$ or $H b_{D}$. Hence, although correctly describing the main trend, Oldroyd's scaling is insufficient to quantitatively capture the location of the yield interface in the configurations studied here. Note also that the larger deviations observed in the experimental case could be due to more complex rheological features of Carbopol. In particular, these deviations may be related to the slight asymmetry of the interface shape, presumably due to viscoelasticity, reported in [8].
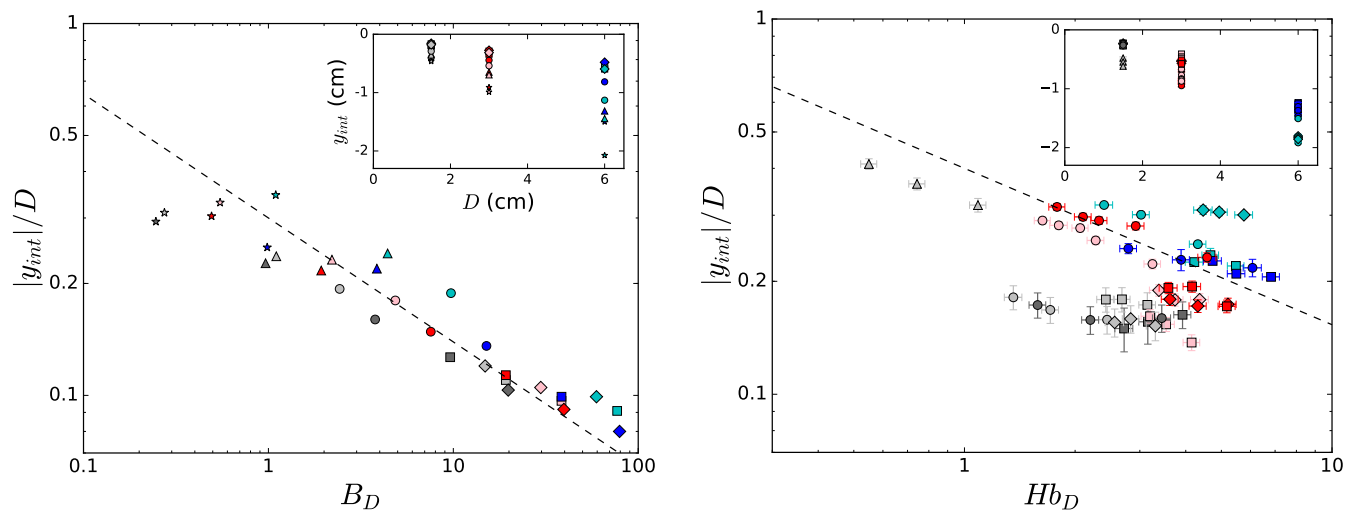

Figure 8: Dead zone interface scaled by cavity length $\left|y_{\text {int }}\right| / D$ as a function of viscoplastic numbers $B_{D}$ or $H b_{D}$ in $\log$ scales (insets: $y_{i n t}$ as a function of $D$ ). Beware of the slightly different vertical scales on both plots. Glyphs correspond to Table 1, while colors legend are indicated in Figure 2. Left: simulations. Dashed line indicates the power law $B_{D}^{-1 / 3}$. Right: experiments. Dashed line indicates the power law $H b_{D}^{-1 /(n+2)}$, with $n=0.4$.

\subsection{Flow characteristics at the boundary layer interface}

We recall that the boundary layer denotes in our case the zone of stress increase observed in the cavity between the dead zone and the quasi-Poiseuille 
flow zone above (see Fig. 6). As shown in Figure 9, a linear relation is found, both in experiments and in simulations, between the velocity $U_{s}$ measured at the boundary layer interface and the plug velocity in the incoming channel $U_{u p}$. Interestingly, the slope of this linear relation appears to be close to 0.4 in both cases. The velocity at boundary layer interface $U_{s}$ thus appears to be essentially controlled by the incoming flow, independently of cavity characteristics.
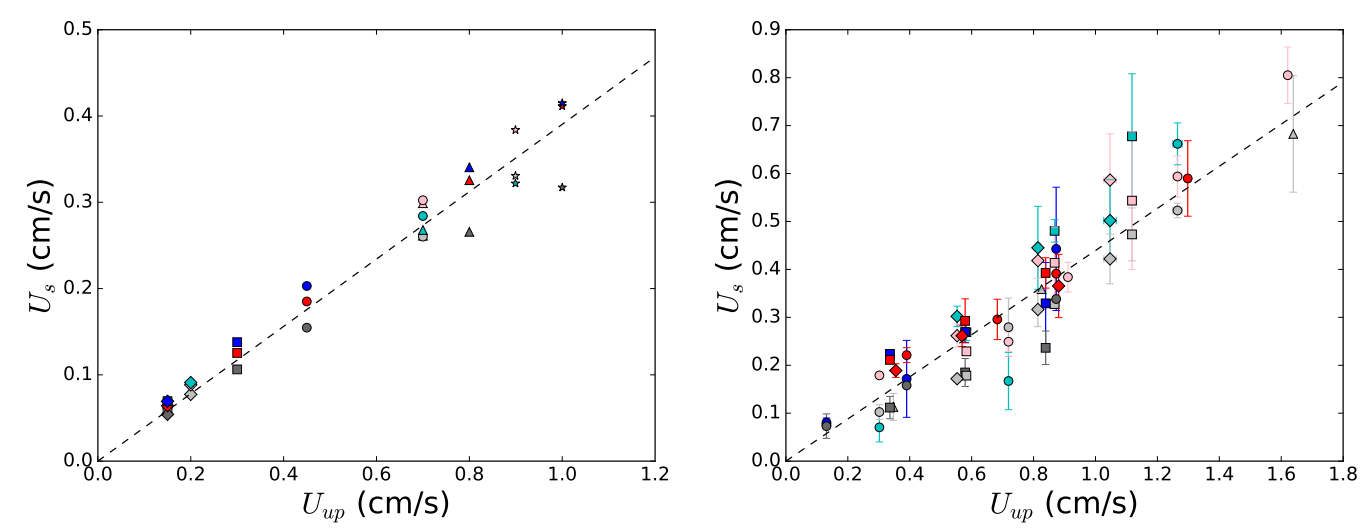

Figure 9: Flow velocity at boundary layer interface $U_{s}$ versus plug velocity in the incoming channel flow $U_{u p}$. Glyphs correspond to Table 1, while colors legend are indicated in Figure 2. Left: simulations (dashed line of slope $\approx 0.39$ ). Right: experiments (dashed line of slope $\approx 0.44)$.

Similarly the maximum stress $\tau_{m}$, which is measured on the boundary layer interface, appears to be directly related to incoming flow characteristics. Figure 10 shows the existence of a linear relation between $\tau_{m}$ and the stress on the wall $\tau_{w}$ in the incoming channel. In both experiments and simulations, the data appear to follow $\tau_{m} \approx 0.8 \tau_{w}$. Furthermore, since the incoming flow obeys a viscoplastic Poiseuille equation, the ratio $\tau_{w} / \tau_{y}$ is itself directly related to the incoming viscoplastic number $B_{u p}$ or $H b_{u p}$. Accordingly, a good correlation is also observed between $\tau_{m} / \tau_{y}$ and $B_{u p}$ or $H b_{u p}$ in both simulations and experiments (Fig. 10).

\subsection{First conclusions}

The results shown above confirm the agreement between the numerical simulations and the experiments, despite the different viscoplastic constitutive laws considered in the two cases. In particular, comparison of the two 

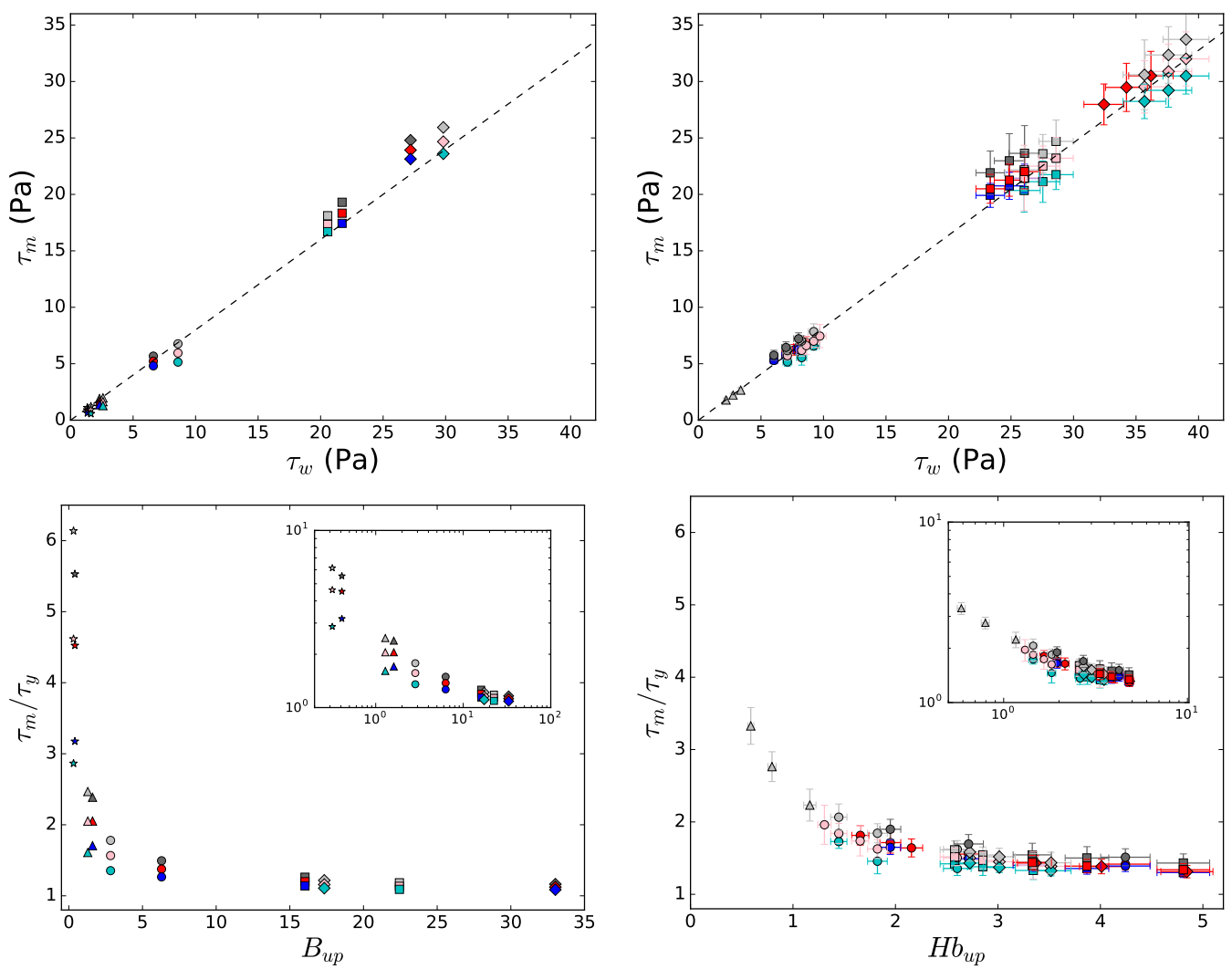

Figure 10: Top: Shear stress at boundary layer interface $\tau_{m}$ as a function of wall shear stress $\tau_{w}$ in the incoming channel. Dashed lines represent the relation $\tau_{m}=0.8 \tau_{w}$. Bottom: Ratio $\tau_{m} / \tau_{y}$ as a function of the viscoplastic number $B_{u p}$ or $H b_{u p}$, with additional log-log representation in inset. Left: simulations. Right: experiments. Glyphs correspondence is indicated in Table 1. For the colors legend, see Figure 2.

data sets allows us to exhibit consistent trends that appear to be characteristic of the flow configuration under study. First, in the range of moderate viscoplastic numbers investigated here, a zoning of the flow in the cavity can be defined by distinguishing between the stress-increase and the stress-decrease layers. Unlike the situation at high values of the viscoplastic number, the shear-rate and shear-stress profiles in these two zones are not symmetric. The stress-decrease zone corresponds to a Poiseuille-like flow layer, while the stress-increase zone delimits a boundary layer in the vicinity of the dead zone. As a further evidence of the asymmetry between these two layers, Figure 11 shows that the thickness of the Poiseuille-like zone $\delta_{P L}$ (measured at $x_{1 / 2}$ ) 
is generally significantly larger than the thickness of the boundary layer $\delta_{B L}$ (except for the longest cavities), again in both experiments and simulations.
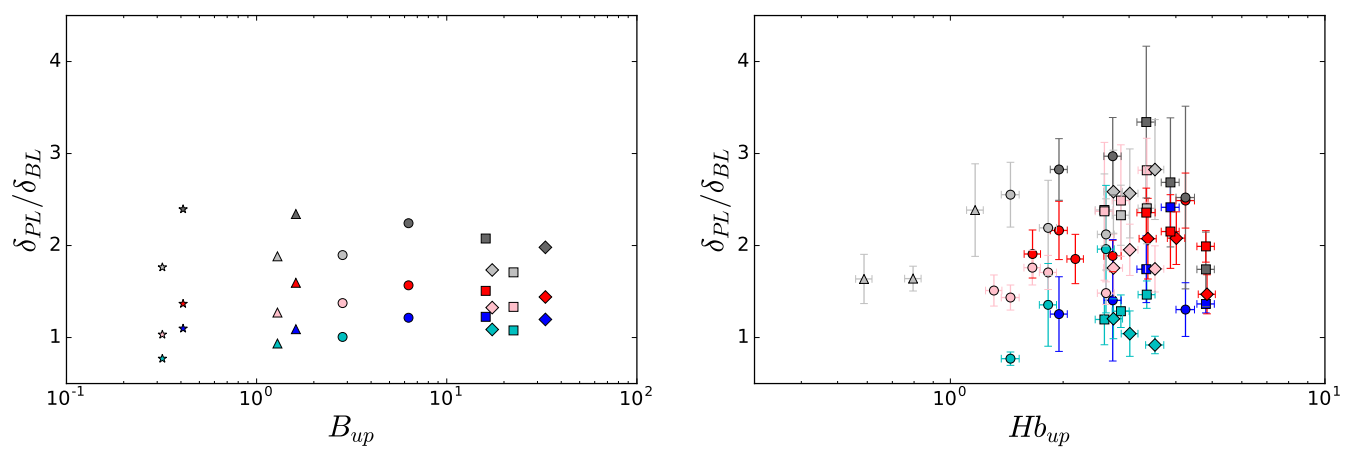

Figure 11: Ratio of Poiseuille-like zone thickness over boundary layer thickness, $\delta_{P L} / \delta_{B L}$ as a function of viscoplastic number $B_{u p}$ or $H b_{u p}$. Left: simulations. Right: experiments. Glyphs correspondence is indicated in Table 1. For the colors legend, see Figure 2.

Second, the location of the dead zone interface $y_{\text {int }}$ appears to be only poorly captured by Oldroyd's scaling, which is expected to hold at high values of the viscoplastic numbers (see section 1). Even if global power-law trends with $B_{D}$ and $H b_{D}$ are visible in the data, a large dispersion is present in both simulations and experiments. Interestingly, however, good correlations are observed between flow velocity and shear stress at the interface of the boundary layer and the characteristics of incoming channel flow (in particular $B_{u p}$ and $\left.H b_{u p}\right)$. Hence, the effective boundary condition felt by the Poiseuillelike flow layer appears to be essentially controlled by the incoming flow, independently of cavity characteristics. In the following, we shall focus in more details on the properties of the boundary layer, with the objective to better identify the factors controlling its extension.

\section{Extended scaling for the boundary layer}

\subsection{Boundary layer equation}

Following the detailed analysis of Balmforth et al. [4], we studied whether Oldroyd's viscoplastic boundary layer equation is verified, at least approximately, in the flow configurations studied here. In the geometry under consideration, Oldroyd's equation writes:

$$
2 \partial_{x} \tau_{x x}+\partial_{y} \tau_{x y}=0
$$


Thanks to the high resolution of our simulations, we can numerically study the derivatives of the stress fields and check how precisely Eq. (4) is verified in the yielded zones. Spatial fields of both terms of Eq. (4) are shown in Figure 12 for a typical example. It is indeed observed that Oldroyd's equation seems to be verified within a few percents in the whole flow zone, and in particular for the profile at the center of the cavity $x_{1 / 2}$ (bottom-right of Fig. 12).
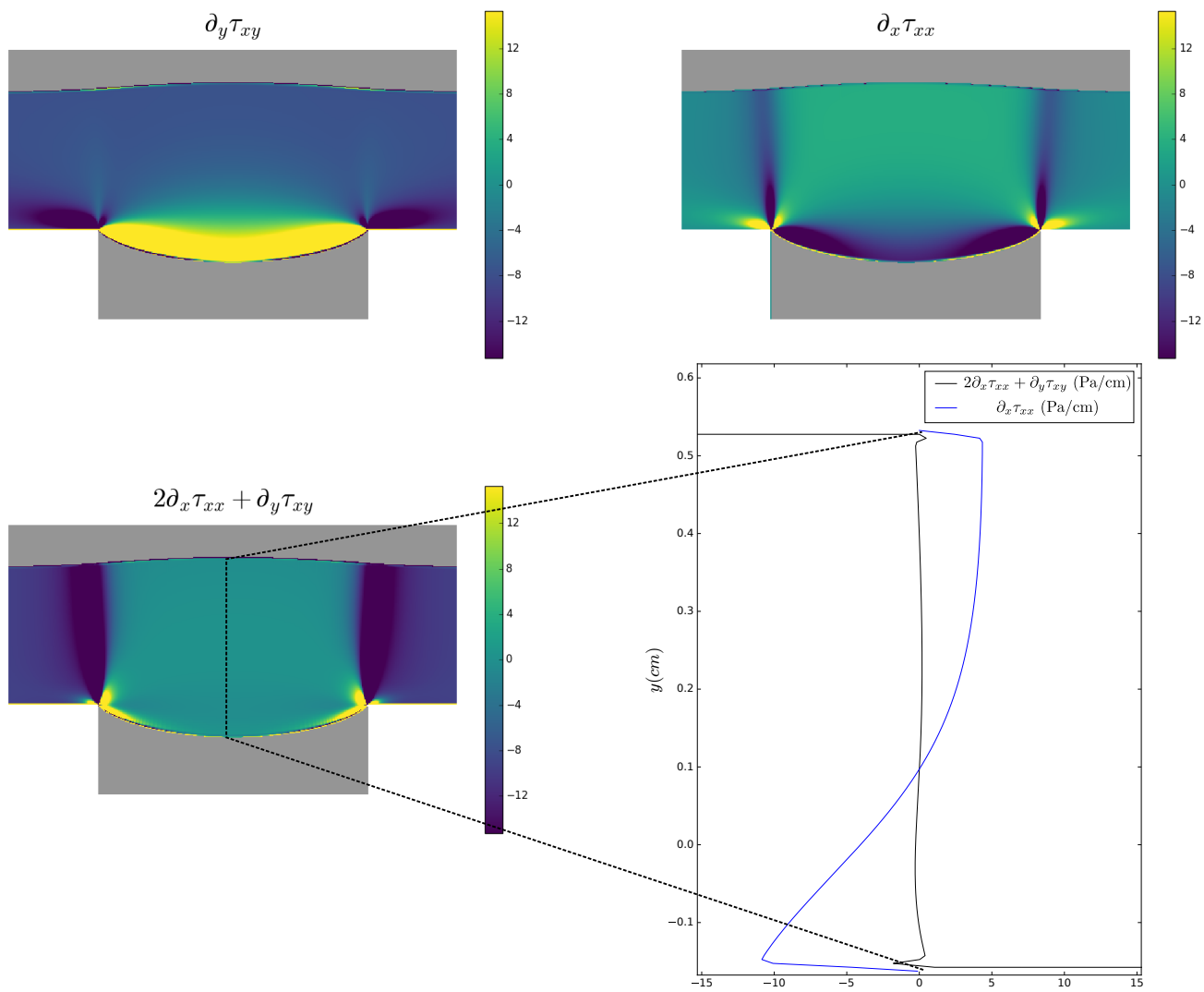

Figure 12: Assessment of Oldroyd's equation from the results of numerical simulations (case $D=1.5 \mathrm{~cm}$ and $h=3 \mathrm{~cm}$ for fluid $\mathrm{F} 4$ ): colormaps of the different terms of the equation, and vertical profiles at $x_{1 / 2}$.

More quantitatively, we systematically compared the $L^{1}$ averages of the 
345

terms of Eq. (4), namely:

$$
\begin{aligned}
L_{\text {Old }}^{1} & =\frac{1}{y_{\text {plug }}-y_{\text {int }}} \int_{y_{\text {int }}}^{y_{\text {plug }}}\left|2 \partial_{x} \tau_{x x}+\partial_{y} \tau_{x y}\right|\left(x_{1 / 2}, y\right) \mathrm{d} y, \\
L_{T x x}^{1} & =\frac{1}{y_{p l u g}-y_{\text {int }}} \int_{y_{\text {int }}}^{y_{\text {plug }}}\left|\partial_{x} \tau_{x x}\right|\left(x_{1 / 2}, y\right) \mathrm{d} y
\end{aligned}
$$

for all the flow configurations simulated. These averages are shown in Figure 13 as a function of the incoming flow viscoplastic number $B_{u p}$. As expected, $L_{\text {Old }}^{1}$ decreases when $B_{u p}$ increases. Observe however that $L_{\text {Old }}^{1}$ remains always significantly smaller than $L_{T x x}^{1}$, and reasonably close to zero, for all the simulations performed. It can thus be concluded that Oldroyd's equation is reasonably verified even in the regimes of moderate Bingham number considered in this study.

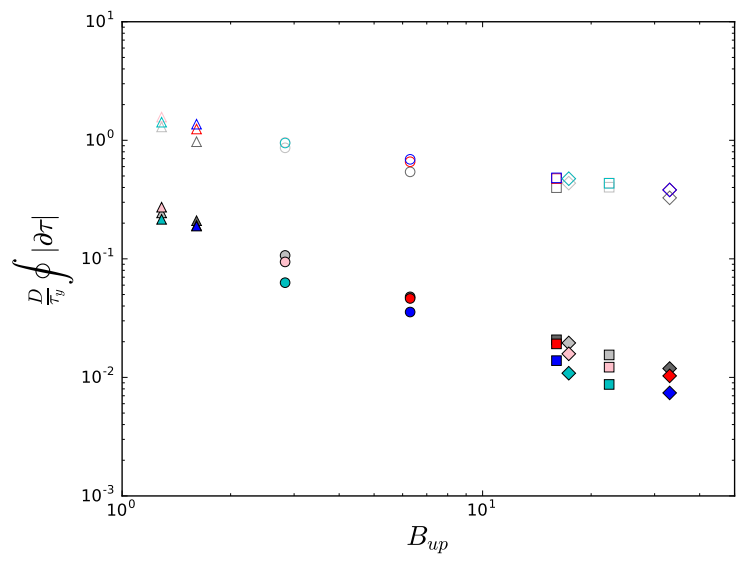

Figure 13: $L^{1}$ averages defined in Eqs. (5) and (6), nondimensionalized by $D / \tau_{y}$, as a function of Bingham number $B_{u p}$ for all simulations performed: $L_{T x x}^{1}$ (with empty glyphs) and $L_{\text {Old }}^{1}$ (with filled glyphs).

\subsection{Generalized scaling for boundary layer thickness}

Previous section showed that the flow zone above the cavity obeys a viscoplastic boundary layer equation even at moderate values of the viscoplastic number. Yet recall that, unlike at high viscoplastic numbers [4], the location of the yield interface $y_{\text {int }}$ is only poorly captured by an Oldroyd's scaling in our results (see Fig. 8). We hypothesize that this apparent paradox is due to the absence of symmetry of the shear-rate and shear-stress profiles in 


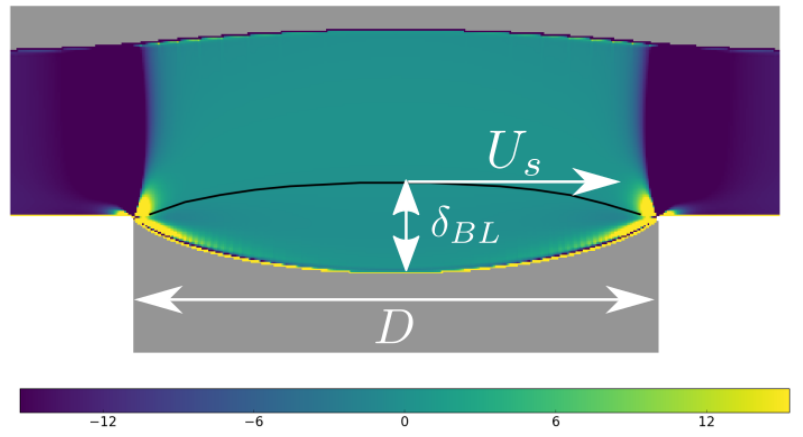

Figure 14: Sketch recalling the definition of the boundary layer considered in this study and the associated characteristic variables used for the new scaling proposed (see text). The upper limit of the boundary layer corresponds to the maximum of the shear stress (black curve). The colormap is the same as in Figure 12(bottom-left): magnitude of the stress derivatives involved in Oldroyd's boundary layer Eq. (4).

the flow zone. We thus checked whether a more consistent scaling emerges when considering only the stress-increase zone, namely the boundary layer defined in our case. Assuming that the proper velocity scale for this layer is the value $U_{s}$ at boundary layer interface (Fig. 14), we defined yet another Bingham number, $B_{\text {cav }}$, based on $U_{s}$ and cavity length $D$ (see Tab. 2). As shown in Figure 15, the normalized thickness $\delta_{B L} / D$ does indeed appear to be univocally related to $B_{\text {cav }}$. All simulated points nicely collapse on a single trend consistent with a generalized Oldroyd's scaling:

$$
\frac{\delta_{B L}}{D} \propto B_{c a v}^{-1 / 3}
$$

Recall that $U_{s}$ was shown to be correlated to incoming flow velocity $U_{u p}$ 355 (Fig. 9). Accordingly, from the above relation between $\delta_{B L}$ and $B_{c a v}$, we could also expect the existence of a relation between $\delta_{B L}$ and the classical Bingham number $B_{D}$ defined on the base of $U_{u p}$ [4]. In practice, however, it appears that the correlation of $\delta_{B L} / D$ with $B_{D}$ (not shown) is far less good than with $B_{\text {cav }}$, suggesting that the observed relation between $U_{s}$ and $U_{u p}$ is only approximate. The Bingham number $B_{c a v}$ appears to represent the best control variable for the boundary layer thickness in our case.

Similarly Figure 16 shows that, in spite of a larger dispersion due to measurement errors, a good correlation is observed in experimental results between $\delta_{B L} / D$ and the Herschel-Bulkley number $H b_{\text {cav }}$ defined on the basis 

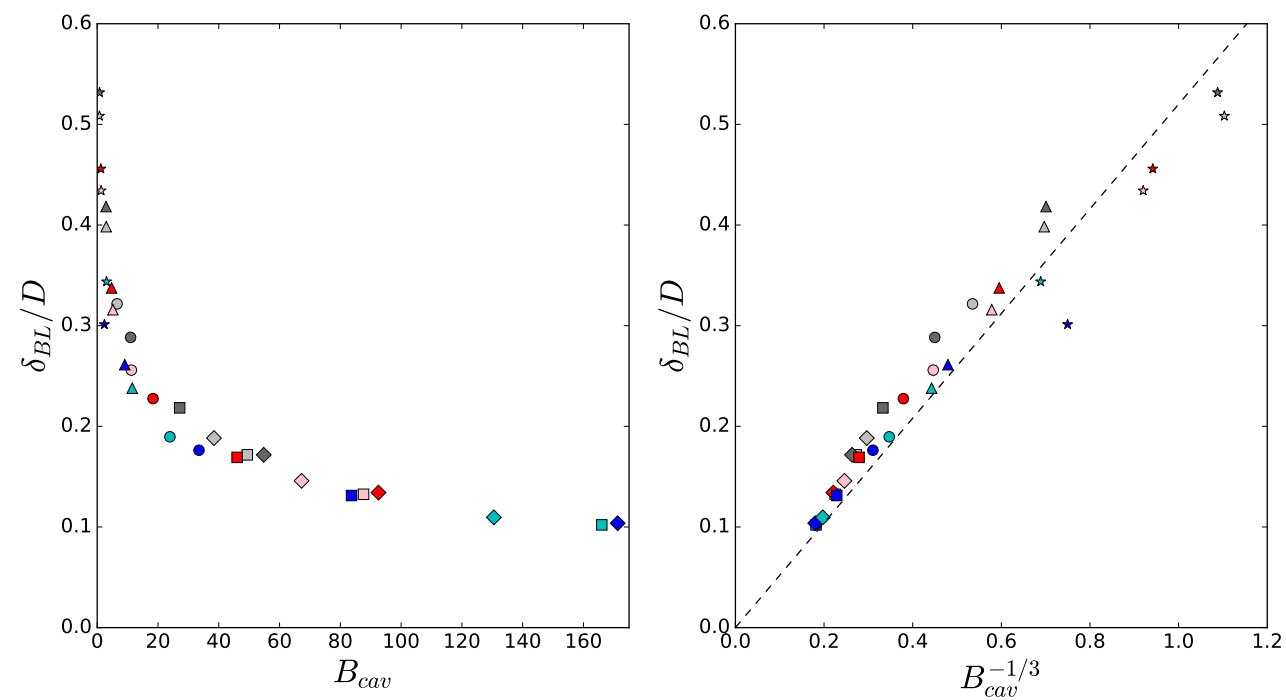

Figure 15: Numerical simulations: evolution of normalized boundary layer thickness $\delta_{B L} / D$ as a function of Bingham number $B_{c a v}$ (left) and $B_{c a v}^{-1 / 3}$ (right). Dashed line has a slope of $\approx 0.52$. Glyphs correspondence is indicated in Table 1 . For the colors legend, see Figure 2.

of $U_{s}$ and $D$ (Tab. 2). Here also, all the data are consistent with HerschelBulkley version of Oldroyd's scaling, which writes:

$$
\frac{\delta_{B L}}{D} \propto H b_{c a v}^{-1 /(n+2)} .
$$

These results justify a posteriori the appellation of boundary layer for the stress-increase zone in our results. Recall that this boundary layer is bounded by an unyielded zone on only one of its sides, and thus typically corresponds to half of the boundary layer defined in [4].

Finally, Figure 17 shows that, unlike $\delta_{B L}$, the thickness of the Poiseuillelike layer $\delta_{P L}$ does not seem to follow an Oldroyd's scaling. Correlations between $\delta_{P L} / D$ and $B_{c a v}$ or $H b_{\text {cav }}$ are relatively poor in both simulation and experimental results. This confirms that, although Oldroyd's equation is also verified, the Poiseuille-like layer does not behave as a boundary layer in the flow regime studied here. Observe nevertheless that correlations between $\delta_{P L} / D$ and $B_{c a v}$ or $H b_{c a v}$ seem to improve for the largest values of the viscoplastic numbers investigated (hence the lowest values of $B_{c a v}^{-1 / 3}$ and $H b_{\text {cav }}^{-1 /(n+2)}$, which may be indicative of a progressive transition towards the 

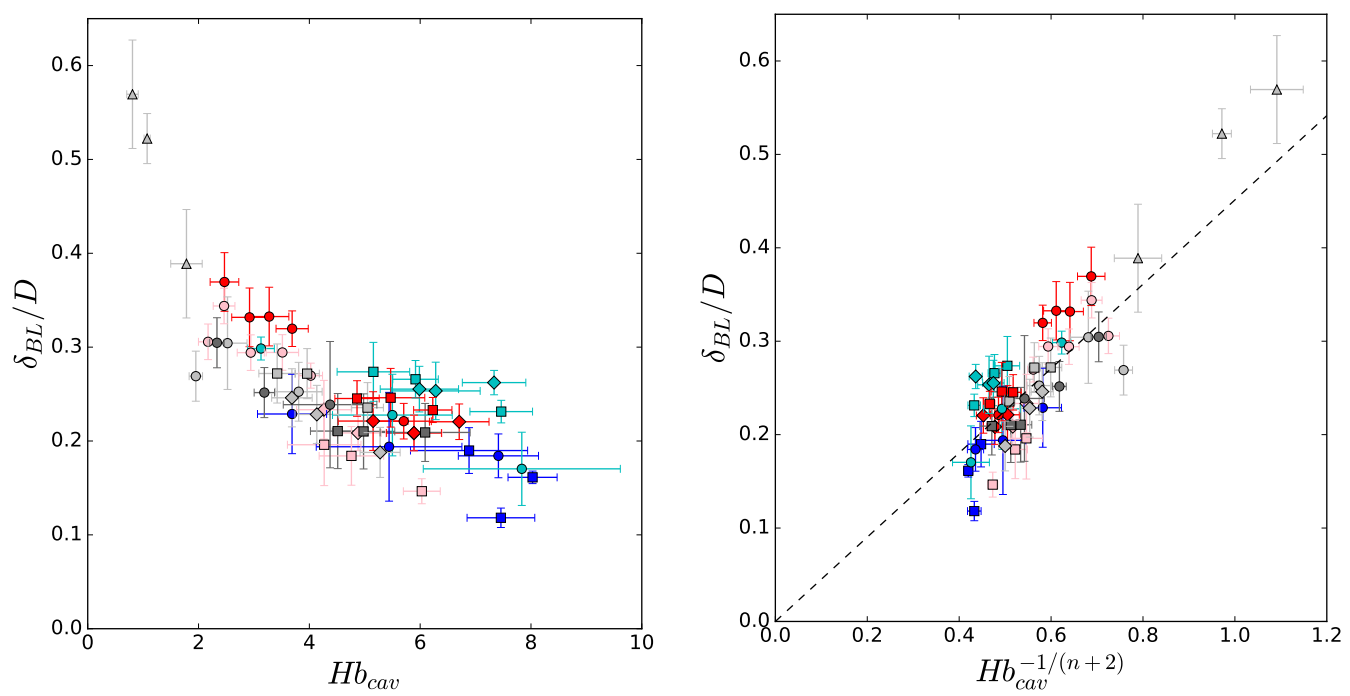

Figure 16: Experimental results: evolution of normalized boundary layer thickness $\delta_{B L} / D$ as a function of Herschel-Bulkley number $H b_{\text {cav }}$ (left) and $H b_{\text {cav }}^{-1 /(n+2)}$ (right). Dashed line has a slope of $\approx 0.45$. Glyphs correspondence is indicated in Table 1 . For the colors legend, see Figure 2.

asymptotic regime in which the whole flow zone behaves as a viscoplastic boundary layer.

\section{Final remarks}

The results obtained in this study lead to generalize the notion of viscoplastic boundary layer previously introduced at high values of the viscoplastic numbers to describe the flow layer forming between two unyielded zones. We show that, at moderate values of the viscoplastic numbers, only the portion of the flow layer in which the shear rate and shear stress increase (see Fig. 14), effectively behaves as a boundary layer. In this case, this boundary layer ensures the transition between the unyielded dead zone that forms in the cavity and the fully-developed, Poiseuille-like flow zone above. Interestingly however, the thickness of this boundary layer is found to follow the same Oldroyd's scaling as that obtained in the asymptotic regime, providing adapted definitions of the viscoplastic numbers are introduced. Note also that this boundary layer actually corresponds to the so-called shear band introduced in [16], with the difference that we provided here a detailed description of the dynamics (velocity and shear-rate profiles) inside this layer. 

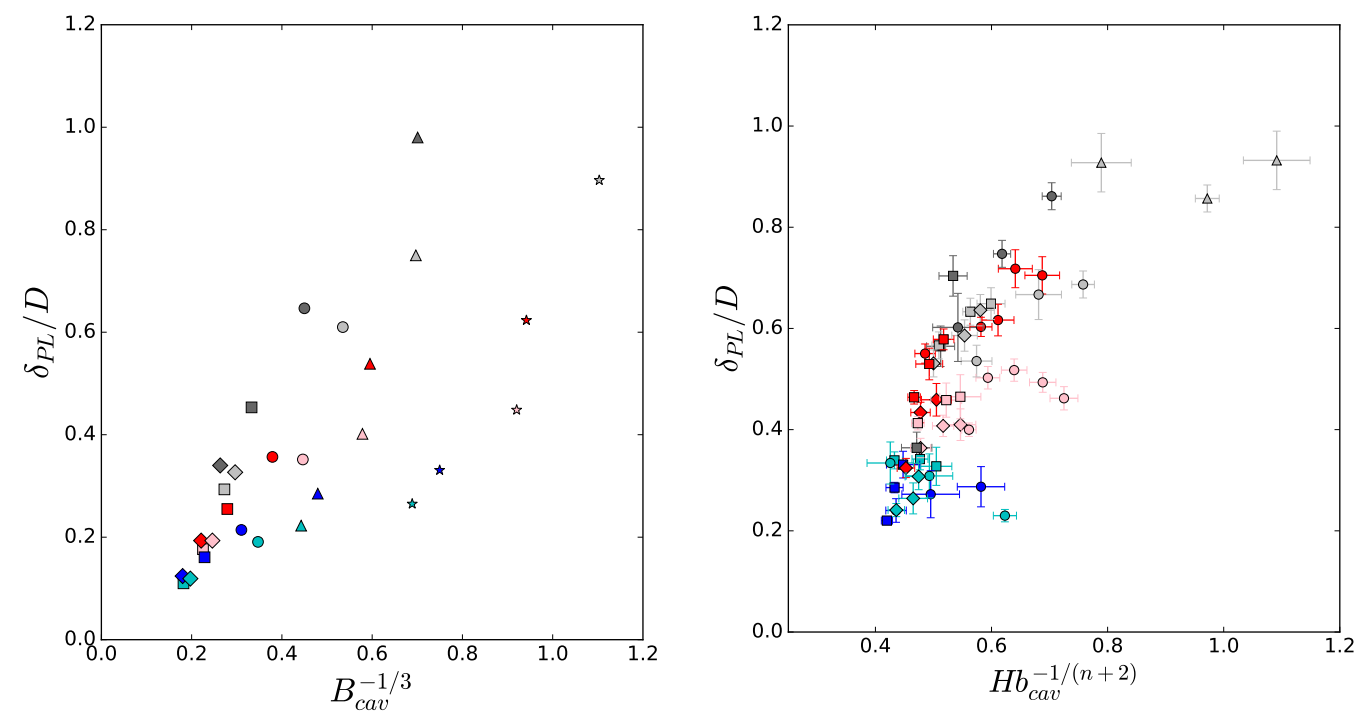

Figure 17: Evolution of normalized Poiseuille-like layer thickness $\delta_{P L} / D$ as a function of viscoplastic number $B^{-1 / 3}$ or $H b_{\text {cav }}^{-1 /(n+2)}$. Left: simulations. Right: experiments. Glyphs correspondence is indicated in Table 1. For the colors legend, see Figure 2.

These results call for an extension of viscoplastic boundary layer theory at smaller values of the viscoplastic numbers $B$ and $H b$. Since the same boundary layer equation appears to be verified, the main difference with the situation at high viscoplastic numbers appears to be the lack of symmetry of the shear-rate profiles in the flow zone. A more accurate description of the velocity and shear-rate profiles would therefore need to be worked out in order to adapt the developments performed in [4]. A proper investigation of the transition towards the asymptotic regime, in which symmetric shear-rate profiles are recovered and the whole flow zone behaves as a boundary layer, also represents an interesting prospect for future work.

Finally, we want to stress that the detailed cross-comparison between experimental and numerical results performed here, constituted a fruitful approach to exhibit features and trends that can be regarded as characteristic of the viscoplastic rheology. In experiments, real yield-stress fluid frequently present additional rheological properties (e.g. viscoelasticity in the case of Carbopol), which, combined with measurement errors, tend to add dispersion to data and blur the trends. Numerical simulations based on finely-resolved meshes and non-regularized schemes do not suffer from this limitation, and provide insights into variables (e.g., stress fields) that are difficult to access 
experimentally. Since the simulations are based on specific viscoplastic constitutive laws, comparisons with experiments remains nevertheless necessary to ensure that the features highlighted numerically, e.g. here the viscoplastic boundary layer, are also visible in the physical world. We argue that such an intertwined dialog between experimental and numerical results represents a promising venue to advance knowledge on the flow of yield-stress materials in complex configurations, notably when coexistence between yielded and unyielded zones is involved.

\section{Acknowledgments}

420 We thank the Referees for their comments which contributed to improve the quality of the article. This work is done within the framework of an INFINITI structuring project of the Mission pour l'Interdisciplinarite of the CNRS, which is here acknowledged. This project, Visco3Plug, was funded by the CNRS, France, in 2017 and 2018. Numerical simulations were done using computer resources of the Pôle Scientifique de Modélisation Numérique (PSMN), Lyon, France.

\section{References}

[1] A. Roustaei, I. A. Frigaard, The occurrence of fouling layers in the flow of a yield stress fluid along a wavy-walled channel, Journal of NonNewtonian Fluid Mechanics 198 (2013) 109-124. 3

[2] A. Roustaei, A. Gosselin, I. A. Frigaard, Residual drilling mud during conditioning of uneven boreholes in primary cementing. Part 1: Rheology and geometry effects in non-inertial flows, Journal of Non-Newtonian Fluid Mechanics 220 (2015) 87-98. 3

[3] A. Marly, P. Vigneaux, Augmented Lagrangian simulations study of yield-stress fluid flows in expansion-contraction and comparisons with physical experiments, Journal of Non-Newtonian Fluid Mechanics 239 (2017) 35-52. doi:10.1016/j.jnnfm.2017.01.002. 3, 4, 7, 9

[4] N. J. Balmforth, R. V. Craster, D. R. Hewitt, S. Hormozi, A. Maleki, Viscoplastic boundary layers, Journal of Fluid Mechanics 813 (2017) 929-954. 3, 4, 12, 15, 18, 20, 21, 22, 24 
[5] P. R. de Souza Mendes, M. F. Naccache, P. R. Varges, F. H. Marchesini, Flow of viscoplastic liquids through axisymmetric expansionscontractions, Journal of Non-Newtonian Fluid Mechanics 142 (2007) 207-217. 3

[6] D. Bonn, M. M. Denn, L. Berthier, T. Divoux, S. Manneville, Yield stress materials in soft condensed matter, Review of Modern Physics 86 (2017) 035005. 3

[7] D. Dall'Onder dos Santos, S. L. Frey, M. F. Naccache, P. R. de Souza Mendes, Flow of elasto-viscoplastic liquids through a planar expansion-contraction, Rheological Acta 53 (2014) 31-41. 3, 6

[8] L. H. Luu, P. Philippe, G. Chambon, Flow of a yield-stress fluid over a cavity: Experimental study of the solid-fluid interface, Journal of NonNewtonian Fluid Mechanics 245 (2017) 25-37. doi:10.1016/j.jnnfm. 2017.04.011. 3, 4, 5, 6, 9, 13, 14, 15

[9] J. G. Oldroyd, Two-dimensional plastic flow of a Bingham solid. A plastic boundary-layer theory for slow motion, Proc. Cambridge Phil. Soc. 43 (1947) 383-395. 4, 12

[10] J. M. Piau, Viscoplastic boundary layer, Journal of Non-Newtonian Fluid Mechanics 102 (2) (2002) 193-218. 4

[11] D. R. Hewitt, M. Daneshi, N. J. Balmforth, D. M. Martinez, Obstructed and channelized viscoplastic flow in a Hele-Shaw cell, Journal of Fluid Mechanics 790 (2016) 173-204. 4

[12] J.-M. Piau, Carbopol gels: Elastoviscoplastic and slippery glasses made of individual swollen sponges. Meso- and macroscopic properties, constitutive equations and scaling laws, Journal of Non-Newtonian Fluid Mechanics 144 (2007) 1-29. 6

[13] G. Chambon, A. Ghemmour, M. Naaim, Experimental investigation of viscoplastic free-surface flows in steady uniform regime, Journal of Fluid Mechanics 754 (2014) 332-364. 6

[14] L. H. Luu, Y. Forterre, Drop impact of yield-stress fluids, Journal of Fluid Mechanics 632 (2009) 301-327. 6 
[15] L.-H. Luu, P. Philippe, G. Chambon, Experimental study of the solidliquid interface in a yield-stress fluid flow upstream of a step, Physical 475 Review E 91 (1) (2015) 013013. 6, 7, 11

[16] T. Chevalier, S. Rodts, X. Chateau, J. Boujlel, M. Maillard, P. Coussot, Boundary layer (shear-band) in frustrated viscoplastic flows, EPL (Europhysics Letters) 102 (4) (2013) 48002. 7, 12, 23

[17] R. Hill, Mathematical Theory of Plasticity, Oxford University Press, 1950. 11 